\title{
Il governo dell'innovazione farmaceutica in Italia: quali strumenti di governance regionale proporre?
}

\author{
Sabina Nuti \\ Laboratorio Management e Sanità, Instituto di Management, Scuola Superiore Sant'Anna, Pisa - Italy
}

\begin{abstract}
Managing pharmaceutical innovation in Italy: which regional governance tools can be adopted?
Within the Italian federalist framework, national and regional governance tools for pharmaceutical care have been developed in recent years. From a financial perspective, the pharmaceutical outpatient expenditure has already been put under control and this has markedly contributed to reducing the overall costs. The hospital pharmaceutical expenses instead have grown. On this last element bears how innovative and expensive drugs are introduced and managed; among these drugs, cancer drugs have a decisive role.

In the last years the AIFA (Agenzia Italiana del Farmaco - Italian Drugs Agency) policies regarding the adoption process of new drugs have stressed the concept of value for money: any innovative and expensive drug is linked to a webbased control register in order to monitor outcomes. The aim is to relate the drug price to the obtained results (payment by result and risk-sharing) or at least to institute simple financial agreements (cost-sharing) that can be defined as managed entry agreements (MEA). The critical point that can cause equity problems is the way these national governance tools are applied in different regional contexts. There are in fact marked differences among Italian regions. Most regions are aware that the only way to rule the system, and in particular the use of innovative drugs, is to have stronger evidence-based management tools able to connect different systems of oncological prescriptions. The aim is to follow patients in different care settings in order to measure the pathway phases in terms of consumption, costs and quality outcomes and therefore evaluate in actual practice the value for money of innovative drugs.
\end{abstract}

Keywords: Governance tools, Oncology, Pharmaceutical innovation

\section{Introduzione}

\section{La razionalizzazione della spesa pubblica e il governo della farmaceutica}

Nel corso dell'ultimo decennio, l'instabilità del quadro macroeconomico ha prodotto profondi cambiamenti nel funzionamento della pubblica amministrazione italiana. L'urgenza di mettere in sicurezza i conti nazionali ha spinto i policy maker a introdurre differenti strumenti di razionalizzazione della spesa pubblica: se da una parte sono stati attivati complessi processi di revisione della spesa, mirati al superamento del criterio storico di finanziamento, dall'altra sono stati definiti tetti di spesa quale strumento gestionale per contenere l'aumento dei costi del sistema.

Accepted: July 22, 2016

Published online: September 27, 2016

Indirizzo per la corrispondenza:

Sabina Nuti

Scuola Superiore Sant'Anna

Piazza Martiri della Libertà, 33

56127 Pisa, Italy

sabina.nuti@sssup.it
Tra i diversi fattori di costo su cui si è concentrata l'azione di governo e di revisione della spesa, grande attenzione è stata rivolta al settore farmaceutico. In particolare, l'Italia si è distinta a livello internazionale per lo sviluppo di un'articolata impalcatura normativa per la regolamentazione dei processi di introduzione sul mercato dei farmaci innovativi e ad alto costo. A partire dalla $\mathrm{L}$. $222 / 2007$, contenente una prima declinazione normativa del concetto di innovatività dei farmaci (1), e dalla successiva definizione dell'algoritmo dell'innovatività ideato da AIFA (Agenzia Italiana del Farmaco) per la concessione dell'Autorizzazione di Immissione al Commercio (AIC), sono stati progressivamente introdotti nuovi strumenti di governo del farmaco. Tra questi, oltre all'introduzione dei budget per famiglie di farmaci e alla definizione di clausole di copertura di eventuali sfondamenti dei tetti, hanno assunto un ruolo di grande importanza due tipologie di strumenti, ossia:

- Managed Entry Agreements (MEA), ossia "accordi di accesso condizionato al mercato per farmaci innovativi e/o ad alto costo" (2), volti a garantire la disponibilità ai pazienti di nuovi trattamenti, attraverso la condivisione del rischio finanziario tra ente pubblico e azienda produttrice.

- $\quad$ Registri dei farmaci, introdotti da AIFA nel 2005 e integrati ufficialmente nel 2013 con il Nuovo Sistema Informativo Sanitario (NSIS), quali strumenti di monitoraggio dell'appropriatezza prescrittiva e di verifica degli stessi MEA. 
All'interno della cornice federalista italiana, agli strumenti nazionali di governance dell'assistenza farmaceutica si sono aggiunti nel tempo altri interventi a responsabilità regionale: dall'abolizione dei prontuari terapeutici regionali (come nel caso di Lombardia, Piemonte, Friuli Venezia Giulia e Marche), all'individuazione di centri autorizzati alla prescrizione di medicinali autorizzati da AIFA, ai criteri di valorizzazione del costo del farmaco per la mobilità con un mix di soluzioni normative e organizzative.

\section{Gli effetti delle politiche di razionalizzazione}

Dall'analisi dell'impatto prodotto dalle politiche di governo della spesa farmaceutica emergono risultati contrastanti. In termini di gestione economica, le diverse leve di governo hanno contribuito positivamente a ridurre la spesa territoriale (da 13,2 miliardi di euro nel 2010 a 11,8 miliardi nel 2014) (3). Sul fronte della farmaceutica ospedaliera, invece, nonostante l'innalzamento del tetto di spesa già nel 2013 (dal 2,4\% al 3,5\% del finanziamento programmato del Fondo Sanitario Nazionale), il monitoraggio della spesa 2014, pari a 4,9 miliardi di euro, ha evidenziato come quasi tutte le Regioni abbiano accumulato un disavanzo di circa 1 miliardo di euro rispetto al livello di finanziamento programmato. La spesa farmaceutica ospedaliera risulta ulteriormente in crescita tra il 2013 e il 2014 (4). Su quest'ultimo dato incide proprio il modello gestionale dei farmaci innovativi e ad alto costo, tra i quali quelli oncologici hanno un peso determinante:

- dei 30 principi attivi a maggiore impatto sulla spesa ospedaliera, ben 12 sono farmaci antineoplastici e immunomodulatori;

- $\quad$ il 92\% (3,9 miliardi di euro) della spesa per farmaci oncologici è gestita dalle strutture pubbliche, assorbendo circa il $40 \%$ della loro spesa farmaceutica complessiva (5).

Spostando il focus sulla dimensione sanitaria, emergono ulteriori punti di attenzione rispetto agli effetti dell'attuale impianto di governance dell'innovazione farmaceutica in Italia. Complice anche il costo estremamente elevato delle nuove terapie, che pone rilevanti questioni di sostenibilità dell'intero sistema sanitario pubblico, l'attuale modello rischia di mettere a rischio l'equità nel diritto di accesso alle cure, sia in termini di confronto transfrontaliero, sia tra le Regioni e intra-regionale.

Così come rilevato nei rapporti dell'Osservatorio sulla condizione assistenziale dei malati oncologici, "analizzando $\mathrm{i}$ dati ufficiali EMA (Agenzia Europea dei Medicinali) ed AIFA in relazione alle tempistiche degli iter registrativi dei farmaci, il tempo medio che intercorre tra l'approvazione europea e la delibera in Italia è oggi di circa 12 mesi, con punte anche di 15 mesi" (6).

Focalizzandosi sull'adozione a livello regionale dei nuovi farmaci antitumorali e ad alto costo, si rilevano forti disparità soprattutto relativamente alle tempistiche nella ricezione delle indicazioni registrate da AIFA.

Con l'accordo Stato-Regioni del 18 novembre 2010, rafforzato poi dalle disposizioni del Decreto Balduzzi del 2012, era stato infatti introdotto per le Regioni l'obbligo di garantire agli assistiti l'immediata disponibilità dei farmaci, inclusi nelle liste AIFA con il requisito dell'innovatività terapeutica di particolare importanza, indipendentemente dall'inserimento nei prontuari regionali (7). A integrazione, con il "decreto del Fare" del 2013 (8), il Governo Letta aveva previsto l'obbligo per I'AIFA di valutare, entro 100 giorni, le domande presentate dalle aziende farmaceutiche per la classificazione e rimborsabilità dei farmaci innovativi approvati dall'EMA.

In sintesi, considerando l'insieme degli interventi regolatori attuati sia sul versante della spesa che sulla dimensione sanitaria, l'attuale modello di governance dell'assistenza farmaceutica ha permesso di conseguire risultati positivi nel contenimento della spesa farmaceutica territoriale che, per tipologia di trattamento, presenta una "più bassa complessità", mentre appare necessaria una più attenta riflessione sull'adeguatezza ed efficacia degli strumenti di governo della componente di spesa farmaceutica ospedaliera. Un'attenta analisi è necessaria, in primo luogo, per tutelare l'equità di accesso alle cure sul territorio nazionale ma anche per aumentare la trasparenza e la chiarezza dei meccanismi di gestione, che sono un presupposto importante per aumentare l'attrattività dell'Italia per gli investimenti in ricerca e sviluppo, su cui la disomogeneità regionale e la lentezza burocratica nel processo autorizzativo possono influire negativamente (9).

\section{La governance dell'innovazione farmaceutica nel contesto italiano}

I livelli e gli attori coinvolti nella governance dell'innovazione farmaceutica sono molteplici e con ruoli diversi nel percorso che prende avvio con la fase dell'autorizzazione al commercio e si conclude con l'effettiva prescrizione del farmaco (Fig. 1). Pur prevedendo un controllo complessivo della spesa farmaceutica mediante l'introduzione di tetti di spesa differenziati per territorio e ospedale, sono previsti interventi specifici per favorire l'introduzione di farmaci innovativi, capaci di garantire risultati più costo-efficaci rispetto ad altre soluzioni già presenti sul mercato.

Una volta conclusa la procedura autorizzativa di un farmaco presso l'EMA, la normativa italiana prevede una corsia preferenziale per tutti i farmaci orfani e di eccezionale rilevanza terapeutica. Il succitato emendamento al decreto Balduzzi che legifera in merito nasce in risposta all'automatica immissione in commercio di farmaci innovativi già autorizzati in Europa, che trovavano automatica collocazione in Fascia $\mathrm{C}$ e cioè a carico del cittadino o del singolo ospedale. Per questa peculiarità, la nuova Fascia era stata ribattezzata "Cnn", ossia Fascia “C non negoziata", pagata a prezzo pieno e quindi estranea a una completa gestione economica e di appropriatezza prescrittiva.

Negli ultimi anni, in questa prospettiva orientata a massimizzare il risultato di esito per ogni euro speso, le politiche di AIFA sull'innovazione farmaceutica hanno tracciato come via maestra per l'appropriatezza della cura quella di legare ogni farmaco innovativo, o ad alto costo, a un registro di controllo web-based. Si prevede che questo, a sua volta, sia legato ad accordi sul prezzo del farmaco condizionati e basati sugli esiti conseguiti (payment by result e risk-sharing) o almeno su semplici accordi finanziari (cost-sharing), che nel complesso vengono definiti come Managed Entry Agreements (MEA) (10). I MEA sono quindi accordi di accesso condizionato al mercato per farmaci innovativi e/o ad alto costo, che consentono di mettere a disposizione nuovi trattamenti per i pazien- 


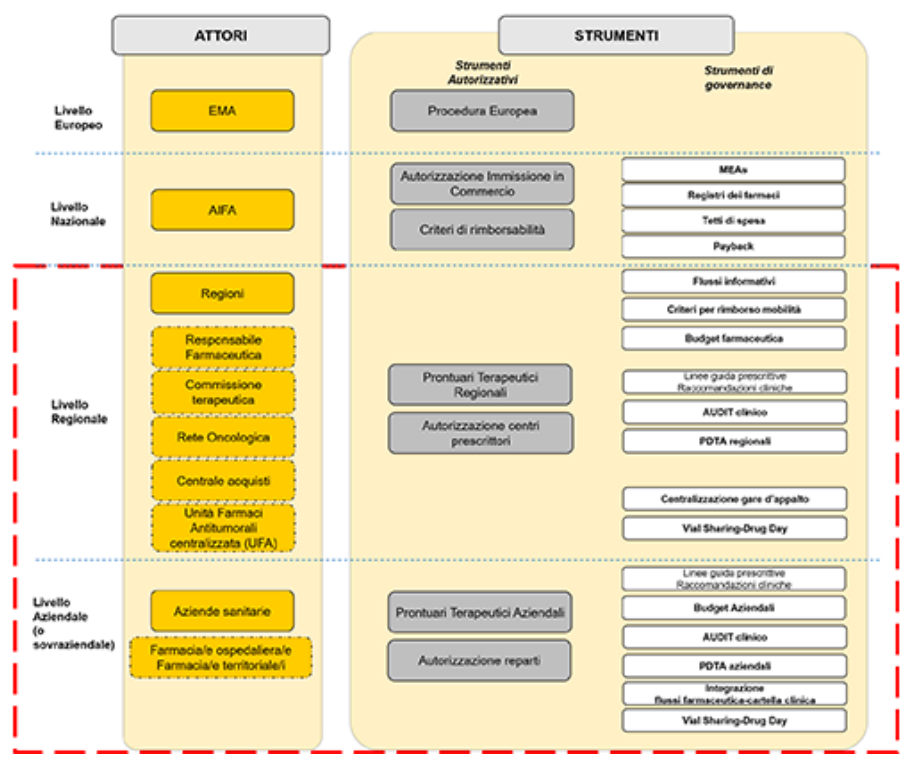

Fig. 1 - Livelli, attori e strumenti per la governance dell'innovazione farmaceutica. Fonte: Laboratorio MeS, Sistema di Valutazione del Network delle Regioni.

ti, pur nell'incertezza data dalla mancanza di informazioni su benefici terapeutici o costi effettivi e rappresentano dei veri meccanismi di governance del sistema sanitario per la gestione dell'assistenza farmaceutica.

In particolare, tutti i registri di monitoraggio dei farmaci, anche quando non legati a uno specifico MEA, possono essere identificati come uno strumento di governance dell'appropriatezza prescrittiva.

Il vero aspetto critico, che può determinare problematiche di equità, sono le modalità con cui questi strumenti effettivamente vengono usati nei diversi contesti regionali.

A livello nazionale, infatti, AIFA, tramite griglie di arruolamento ben definite, i follow-up e le schede di prescrizione, propone strumenti capaci di garantire che pazienti con le stesse caratteristiche biologiche abbiano, almeno formalmente, uguale accesso all'innovazione farmaceutica in tutta Italia. In ambito oncologico, per esempio, viene chiaramente identificato quale "paziente tipo" possa accedere a una nuova terapia (come, ad esempio, per le nuove target therapy) (11); il paziente che ne può beneficiare dovrebbe avere determinate caratteristiche, ossia la presenza o meno di quel recettore o quella proteina espressa sulla cellula neoplastica, che rende automaticamente il paziente eleggibile o meno al trattamento.

Con le suddette indicazioni, tutti i nuovi farmaci in oncologia dovrebbero trovare disponibilità nelle Regioni tramite il Registro AIFA. Alle Regioni viene demandato il compito di identificare, sul proprio territorio, i centri abilitati alla prescrizione. Un primo punto meritevole di approfondimento è su quali basi vengono identificati i centri prescrittori e quali siano gli strumenti a disposizione per verificare il corretto utilizzo delle nuove terapie da parte delle strutture autorizzate.

Il tema degli strumenti di governance che le Regioni possono adottare per garantire da un lato il governo della spesa per l'assistenza farmaceutica, dall'altro l'innovazione e la sperimentazione di nuovi farmaci che migliorino gli esiti e al qualità delle cure dei pazienti diventa quindi un punto cruciale per il conseguimento stesso della missione del sistema sanitario pubblico.

\section{Quali strumenti per migliorare il governo dell'assistenza farmaceutica in oncologia nel contesto regionale?}

Storicamente la spesa farmaceutica regionale è stata sempre scomposta in spesa ospedaliera e territoriale. Nella spesa farmaceutica ospedaliera rientrano i farmaci erogati durante il regime di ricovero, di Day Hospital, ambulatorio terapeutico, mentre nella spesa farmaceutica territoriale rientrano tutti i farmaci erogati dalle farmacie ospedaliere territoriali o distribuiti per conto.

Buona parte delle Regioni ha oramai acquisito una reale maturità nel gestire la spesa territoriale: esempi sono le gare di acquisto centralizzate, la dispensazione per conto e l'introduzione di ticket sui brand per incentivare il consumo dei farmaci generici.

Assai più complessa appare invece la situazione relativa al governo regionale della spesa per i farmaci oncologici. All'interno di un quadro normativo e gestionale altamente complesso, infatti, i farmaci oncologici, in particolar modo quelli innovativi, risultano essere una delle maggiori voci di spesa per i sistemi sanitari regionali. I dati forniti dall'Osmed (Osservatorio Nazionale sull'Impiego dei Medicinali) per i primi mesi del 2015 presentano un quadro che registra un incremento significativo della spesa rispetto al 2014, proprio per questa tipologia di farmaci. Gli anticorpi monoclonali, che sono la principale classe di spesa all'interno del gruppo $\mathrm{L}$, hanno registrato un incremento rispetto al 2014 di ben il 25,4\%. Ciò è dovuto all'immissione in commercio dei nuovi farmaci per il tumore della mammella trastuzumab emtansine e pertuzumab, ossia farmaci approvati in Italia nella seconda metà del 2014, ma esplosi come consumi nel 2015

Un notevole incremento di spesa si è riscontrato anche nel gruppo degli L02 dovuto ai nuovi farmaci per il tumore della prostata, enzalutamide e abiraterone (antagonisti ormonali $+71,8 \%$, antiandrogeni $+>100 \%$ ) (12).

Martini et al, evidenziano come, nelle previsioni di mercato per aree terapeutiche, i farmaci oncologici nel prossimo 
triennio vedranno una crescita media nelle vendite di circa $8 \%$ (13) conquistando un peso sempre maggiore in termini di quote di mercato.

L'introduzione di un numero sempre più considerevole di farmaci innovativi a livello ospedaliero porta le Regioni a muoversi in un contesto sempre più complesso, tale da non permettere una approfondita riflessione sulla gestione delle nuove terapie e sui numerosi accordi negoziali (MEA) a cui i nuovi farmaci sono quasi sempre correlati.

A conferma di questo si sottolinea che ad oggi sono attivi più di 126 registri AIFA, di cui 77 legati ad accordo di risk-sharing (14), mentre le Regioni fino ad ora hanno fatto riferimento a strumenti di governo della spesa farmaceutica basati su database amministrativi del tutto inappropriati per gestire una partita così importante. In particolare, risulta ancora inadeguato il processo di misurazione a confronto tra le Regioni e le Aziende, pur se negli ultimi anni è stato attivato un percorso volto a perseguire questo risultato. Dal 2008 infatti un network di Regioni italiane (15 nel 2015) condivide un sistema di valutazione della performance, promosso dal Laboratorio Management e Sanità della Scuola Superiore Sant'Anna di Pisa, che rappresenta un riuscito esperimento di "valutazione dal basso", di assunzione di responsabilità da parte delle Regioni rispetto alle performance dei propri sistemi sanitari e dunque dei servizi offerti ai propri cittadini. In questa prospettiva, le Regioni non sono il mero oggetto del processo di monitoraggio, quanto i soggetti attivi, coinvolti nel disegno e nello sviluppo dello strumento stesso. Il Sistema di Valutazione ribalta la logica classica della valutazione, perché non nasce con l'intento di valutare l'operato delle Regioni, quanto piuttosto con quello di fornire loro uno strumento utile alla programmazione, alla pianificazione e alla governance dei propri sistemi sanitari. In questa logica, le Regioni scelgono di aderire a una comune piattaforma di gestione dati $(15,16)$, per confrontarsi sulla base del calcolo di un set di indicatori di performance condivisi.

Il Sistema si basa su un principio di adesione volontaria da parte delle Regioni. La scelta degli indicatori e degli standard di valutazione è il frutto di un processo condiviso di discussione e collaborazione tra gli stessi soggetti valutati (le Regioni), sulla base delle proposte avanzate dal Laboratorio MeS (Scuola Sant'Anna) o da parte delle Regioni stesse.

Nel 2015 gli indicatori che compongono il Sistema di Valutazione delle Regioni sono circa 200. Ogni Regione ha invero la possibilità di inserire indicatori specifici, volti a esplorare particolari aspetti che siano rilevanti per il perseguimento di strategie territoriali e non necessariamente di interesse per gli altri soggetti. Ogni Regione ha inoltre la facoltà di calcolare gli indicatori con la granularità che ritiene più utile ai fini della governance regionale: livello regionale, aziendale, area vasta, stabilimento, distretto/comprensorio.

Gli indicatori sono calcolati e condivisi con cadenza annuale, per quanto pressoché tutte le Regioni provvedano a monitorare il loro andamento con frequenza infra-annuale.

La definizione degli indicatori abbraccia quella che può essere definita una prospettiva "gestionale": sono selezionati non tanto perché capaci di fotografare in dettaglio la situazione epidemiologica dei contesti regionali, quanto perché in grado di monitorare processi e soluzioni organizzative degli attori del Sistema: in altri termini, sono inclusi quegli indicatori che siano ritenuti utili alle scelte organizzative. Il protocollo di calcolo degli indicatori è inoltre definito in modo tale da permettere alle aziende stesse del network di calcolare tempestivamente il dato, di aggiornarlo e monitorarne regolarmente l'andamento, di integrarlo nei meccanismi aziendali di programmazione e controllo (segnatamente, nei processi di budgeting).

Per quanto riguarda il governo dell'assistenza farmaceutica, il network delle Regioni ha condiviso un set di indicatori focalizzato soprattutto, sia in termini di spesa che di appropriatezza ed efficienza prescrittiva, sulla farmaceutica territoriale. Questa scelta è dovuta in buona misura all'attuale disponibilità di dati confrontabili tra Regioni, basata sui flussi amministrativi della farmaceutica convenzionata.

Relativamente alla spesa per i farmaci oncologici, l'unico indicatore fino ad oggi considerato dal network delle Regioni (17) è stato quello relativo alla spesa media per paziente per i farmaci distribuiti dalla strutture ospedaliere e territoriali in erogazione diretta e per conto (Fig. 2). Il limite, ad oggi, di tale indicatore è quello di aver tralasciato dal computo la spesa per le formulazioni endovena preparate e somministrate dalle Oncologie all'interno delle strutture ospedaliere.

L'indicatore ha solo finalità di osservazione e nella sua attuale formulazione si presenta come incompleto e incapace di misurare sia il costo sostenuto in media per i pazienti oncologici per i nuovi farmaci, sia l'eventuale problematica di accesso e variabilità evitabile tra utenti residenti in diverse aree geografiche o curati da diversi centri di erogazione delle cure.

La prospettiva integrata ospedale-territorio appare quindi essenziale per una corretta valutazione della spesa, ma non solo. L'analisi dei dati lungo il percorso di cura permette di tracciare non solo i costi ma anche di monitorare l'efficacia complessiva di un trattamento. Emanciparsi dalla visione del mero "costo per terapia" permette di apprezzare appieno le opportunità offerte dall'innovazione farmaceutica nel ridisegnare i percorsi assistenziali. Gli indicatori di monitoraggio e di valutazione sono infatti utili nella misura in cui concorrono ad attivare percorsi di miglioramento della pratica assistenziale e questo è possibile solo se si concentrano su fenomeni che siano al tempo stesso rilevanti e manovrabili. In merito al primo punto, gli indicatori devono rilevare il costo e l'efficacia della gestione assistenziale del percorso oncologico, ossia, per esempio, indagare se l'adozione di farmaci apparentemente più costosi si traduca in un risparmio nel medio o nel lungo periodo. In merito al secondo punto, alla manovrabilità, un indicatore è utile nella misura in cui va a fornire un'informazione rilevante a quei professionisti che siano effettivamente responsabili del risultato e che quindi possano azionare le leve per migliorare i risultati stessi.

La sfida dei prossimi anni sarà quindi l'implementazione di flussi ospedalieri dettagliati e integrati rispetto a quelli territoriali, con il superamento di un monitoraggio della spesa ospedaliera a indirizzo quasi esclusivo interno alla struttura o al più all'azienda. È tutt'oggi paradossale che le Direzioni delle strutture ospedaliere non abbiano accesso alle informazioni sulle proprie prescrizioni di farmaci oncologici ad alto costo, quando si tratta di formulazioni che il paziente ritira sul territorio con prescrizione a registro AIFA, 


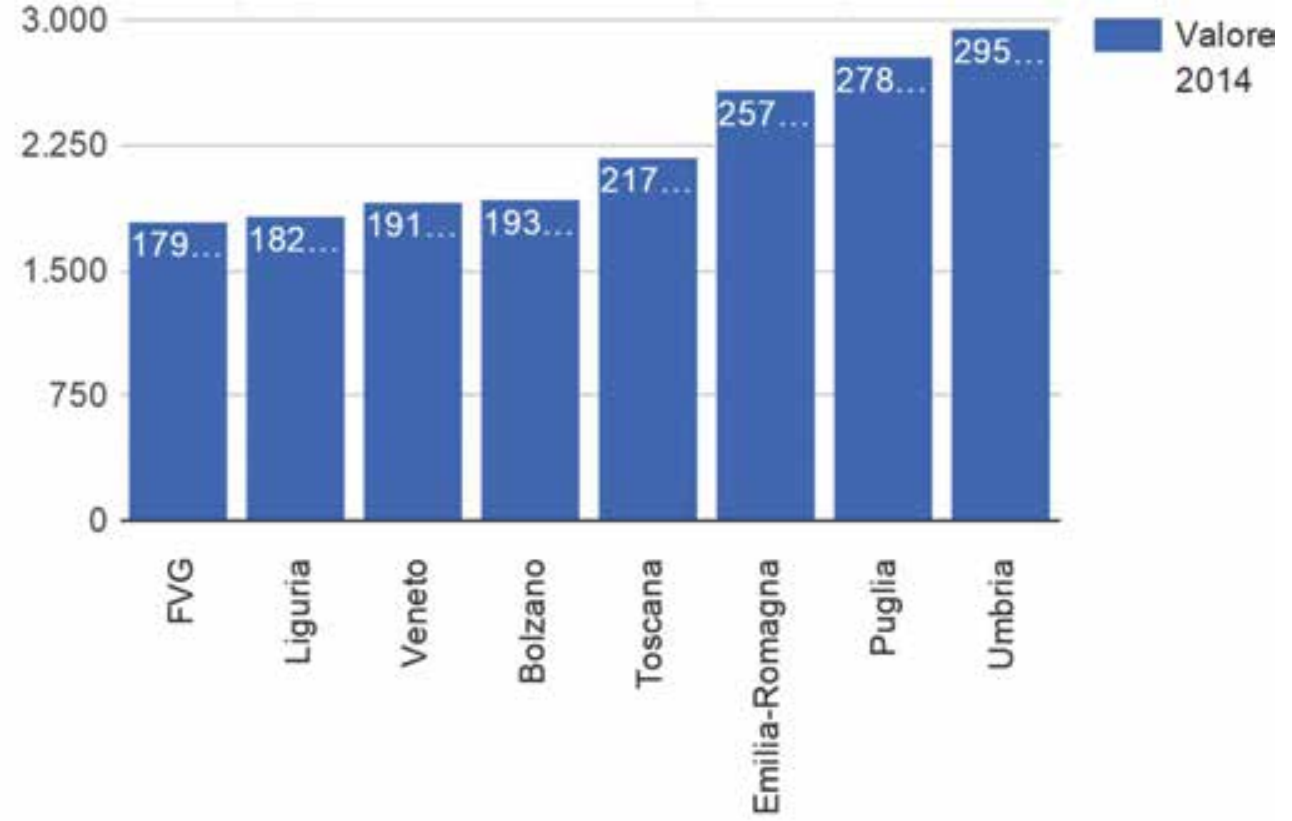

Fig. 2 - Indicatore Spesa media per paziente trattato con farmaci oncologici (in distribuzione diretta e per conto). Fonte: Laboratorio MeS, Sistema di Valutazione del Network delle Regioni. pur avendo la totale responsabilità di queste prescrizioni.

L'integrazione tra database ospedalieri e territoriali permetterà un salto di qualità importante: da una gestione prevalentemente settoriale a una integrata e di sistema. In questa prospettiva, potranno essere sviluppati indicatori di performance farmaceutica capaci di evidenziare la trasversalità dell'impatto, diretto e indiretto sui livelli di ospedalizzazione e di cura della popolazione, sull'uso appropriato ed efficiente della tecnologia "farmaco". Sta infatti crescendo, perlomeno in alcune Regioni, la consapevolezza che l'unico modo per governare il sistema, e in particolare l'utilizzo dei farmaci innovativi, sia quello di dotarsi di database clinici comunicanti con i vari sistemi di prescrizione oncologica. L'obiettivo è seguire con il sistema di rilevazione i pazienti nei diversi setting assistenziali per ricostruire sistematicamente le tappe del loro percorso in termini di consumi, costi ed esiti. Misurare i risultati di outcome è essenziale per affrontare l'innovazione farmaceutica non più come un costo ma come un'opportunità per migliorare le cure, prima in via sperimentale e pilota, sotto il controllo dei centri autorizzati, e poi, in caso di significativo valore aggiunto per ogni euro speso, diffusa a tutti gli aventi diritto, senza disparità.

\section{Conclusioni}

La complessità nella regolamentazione e gestione dell'innovazione nel settore farmaceutico pone I'Italia di fronte alla priorità di identificare una formula tecnico-organizzativa che permetta contemporaneamente di preservare I'universalità del diritto alla salute e la sostenibilità economica del SSN (Servizio Sanitario Nazionale) ma anche di aumentare l'attrazione di investimenti in ricerca e sviluppo sul territorio nazionale con strategie di condivi- sione dei rischi con i produttori.

Pur se si riconosce una volontà sempre più accentuata di centralizzazione delle decisioni relativamente alla governance dei nuovi farmaci oncologici tramite la gestione AIFA, appare urgente mappare i modelli attualmente adottati dalle Regioni nella gestione locale per misurare i gap di equità e i differenziali esistenti nel trattamento riservato a utenti residenti in diverse Regioni e in cura presso centri diversi.

A tal fine sarebbe opportuno monitorare le modalità con cui si svolge nei diversi contesti regionali il processo di cura in oncologia, se siano stati condivisi dai professionisti Percorsi Diagnostici Terapeutici Assistenziali (PDTA) specifici, quali sia il grado di diffusione delle reti oncologiche, come operino le unità UFA (Unità Farmaci Antitumorali) centralizzate nonché l'utilizzo della preparazione farmaceutica in condivisione con la rete (vial-sharing) e delle funzioni di HTA (Health Technology Assessment) e/o di gruppi di lavoro di valutazione del farmaco in seno alle Commissioni Terapeutiche Regionali.

Alla luce di tali informazioni sarà utile rendere disponibili e trasparenti le informazioni di costo e di esito per tutte le fasi del percorso oncologico a tutti i portatori di interesse del sistema sanitario, in primis dei professionisti sanitari, per valutare la bontà delle soluzioni organizzative e gestionali adottate. L'obiettivo è far sì che la valutazione di costoefficacia non sia declinata solo nella prospettiva del singolo paziente ma in termini di salute della popolazione (population based) (18).

Il riferimento alla "popolazione" e non ai "pazienti" chiama lo stesso settore della farmaceutica a un rinnovato sforzo di public accountability: il processo di valutazione di costoefficacia è infatti l'infrastruttura informativa sulla quale impiantare un processo di riallocazione delle risorse, sia in 
seno al settore sanitario sia tra i diversi settori della Pubblica Amministrazione, finalmente giustificato da evidenze scientifiche e non dai rapporti di potere dei professionisti o da spinte messe in atto dalle aziende farmaceutiche o ancora determinate da scelte dei policy makers basate sulla ricerca del consenso di breve periodo.

\section{Disclosures}

Financial support: No financial support was received for this submission.

Conflict of interest: The author has no conflict of interest with this submission.

\section{Bibliografia}

1. Fonte AIFA, Agenzia Italiana del Farmaco: Criteri di valutazione per l'attribuzione del grado di innovazione terapeutica. http:// www.agenziafarmaco.gov.it/it/content/criteri-di-valutazione. Ultimo accesso: 1 marzo 2016.

2. Fonte AIFA, Agenzia Italiana del Farmaco: Managed Entry Agreements: I'AIFA ospita un confronto europeo su scenario e sviluppi futuri. http://www.agenziafarmaco.gov.it/it/content/ managed-entry-agreements-I\%E2\%80\%99aifa-ospita-un-confronto-europeo-su-scenario-e-sviluppi-futuri. Ultimo accesso: 1 marzo 2016.

3. Rapporto Osmed 2014. Spesa farmaceutica territoriale: confronto 2010-2014. Il dato è dato dalla somma tra la spesa convenzionata netta e la spesa per DD e DPC dei farmaci di Classe A. Tabella 5.1.1.

4. Rapporto Osmed 2014. Governo della spesa farmaceutica, p. 75.

5. Rapporto Osmed 2014. Composizione della Spesa farmaceutica complessiva 2014 per I livello ATC e classe di rimborsabilità. Tabella. 6.1.

6. Subito disponibili i farmaci salvavita. Documento AIOM-SIEFAVO, gennaio 2014 .
7. D. L. $158 / 2012$, n. 158 , capo III. Disposizioni in materia di farmaci, comma 2 e 3.

8. D. L. 69/2013, articolo 44, comma 5.

9. Jommi C, Paruzzolo S. Public administration and R\&D localisation by pharmaceutical and biotech companies: a theoretical framework and the Italian case study. Health Policy 2007; 81:117-30.

10. Ferrario A, Kanavos P. Final report prepared "Managed entry agreements for pharmaceuticals: The European experience". AIFA and The London School of Economics and Political Science. April 2013.

11. Kelly CM, Buzdar AU. Using multiple targeted therapies in oncology: considerations for use, and progress to date in breast cancer. Drugs 2013;73(6):505-15.

12. Rapporto Osmed gennaio-settembre 2015. Spesa e consumi nei primi 9 mesi del 2015 per farmaci erogati dalle strutture pubbliche: categorie terapeutiche per I livello ATC. Tabella 15.

13. Martini N, Jommi C, Labianca R, et al. Un nuovo modello di governance per il market access dei nuovi farmaci in oncologia. Una risposta alla complessità e alle sfide del futuro. Forum Service Editore srl, 2015.

14. Fonte AIFA, Agenzia Italiana del Farmaco. Lista aggiornata dei Registri e dei Piani Terapeutici web-based http://www.agenziafarmaco.gov.it/it/content/lista-aggiornata-dei-registri-e-deipiani-terapeutici-web-based. Ultimo accesso: 1 marzo 2016.

15. Network delle Regioni; piattaforma di condivisione dati: http://performance.sssup.it/netval/start.php. Ultimo accesso: marzo 2016.

16. Nuti S, Vola F, Bonini A, Vainieri M. Making governance work in the healthcare sector: evidence from a "natural experiment" in Italy. Health Economics, Policy And Law, 2015.

17. Nuti S, Vola F, Amat P. Sistema di valutazione della performance dei sistemi sanitari regionali: Basilicata, Calabria, Emilia-Romagna, Friuli Venezia Giulia, Lazio, Liguria, Lombardia, Marche, P.A. Bolzano, P.A. Trento, Puglia, Sardegna, Toscana, Umbria, Veneto. REPORT 2014, ISBN 978-884674389-3.

18. Gray JAM. The shift to personalised and population medicine. Lancet 2013;382(9888):200-1. 